\title{
The Early Effect of the Roux-en-Y Gastric Bypass on Hormones Involved in Body Weight Regulation and Glucose Metabolism
}

\author{
Francesco Rubino, MD, * Michel Gagner, MD, FACS, $\ddagger$ Paolo Gentileschi, MD, $\S$ Subhash Kini, MD, $\S$ \\ Shoji Fukuyama, MD,, John Feng, MD, $\S$ and Ed Diamond, MD†
}

\begin{abstract}
Objective: To evaluate the early effect of Roux-en-Y (RYGB) gastric bypass on hormones involved in body weight regulation and glucose metabolism.

Significant Background Data: The RYGB is an effective bariatric procedure for which the mechanism of action has not been elucidated yet. Reports of hormonal changes after RYGB suggest a possible endocrine effect of the operation; however, it is unknown whether these changes are the cause or rather the effect of surgically induced weight loss. We speculated that if the mechanism of action of the RYGB involves an endocrine effect, then hormonal changes should occur early after surgery, prior to substantial body weight changes.
\end{abstract}

Methods: Ten patients with a mean preoperative body mass index (BMI) of $46.2 \mathrm{~kg} / \mathrm{m}^{2}\left(40-53 \mathrm{~kg} / \mathrm{m}^{2}\right)$ underwent laparoscopic RYGB. Six patients had type 2 diabetes treated by oral hypoglycemic agents. Preoperatively and 3 weeks following surgery, all patients were tested for fasting glucose, insulin, glucagon, insulinlike growth factor 1 (IGF-1), leptin, gastric inhibitory polypeptide (GIP), glucagon-like peptide-1 (GLP-1), cholecystokinin (CCK), adrenocorticotropic hormone (ACTH), corticosterone, and neuropeptide Y (NPY).

Results: Changes in mean BMI were rather minimal $\left(43.2 \mathrm{~kg} / \mathrm{m}^{2}\right.$; $P=$ not significant), but there was a significant decrease in blood glucose $(P=0.005)$, insulin $(P=0.02)$, IGF-1 $(P<0.05)$, leptin $(P=0.001)$, and an increase in ACTH levels $(P=0.01)$. The other hormones were not significantly changed by surgery. All the 6 diabetic patients had normal glucose and insulin levels and did not require medications after surgery. The RYGB reduced GIP levels in

From the *IRCAD-EITS, Strasbourg, France and †Department of Endocrinology, Mount Sinai Medical Center, New York, New York. †ेDepartment of Surgery, Weill-Cornell College of Medicine, New York, NY; and §Division of Laparoscopic Surgery, Mount Sinai.

Data presented at the 2002 Annual Meeting of the Society for Surgery of the Alimentary Tract; May 19-22, 2002; San Francisco, California.

Reprints: Michel Gagner, MD, FRCSC, FACS, Weill-Cornell College of Medicine, New York-Presbyterian Hospital, 525 East 68th Street, Box 294, New York, NY 10021. E-mail: mig2016@med.cornell.edu.

Copyright (C) 2004 by Lippincott Williams \& Wilkins

ISSN: 0003-4932/04/24002-0236

DOI: $10.1097 / 01$. sla.0000133117.12646.48 diabetic patients $(P<0.01)$, whereas no changes in GIP levels were found in nondiabetics.

Conclusions: Roux-en-Y gastric bypass determines considerable hormonal changes before significant BMI changes take place. These results support the hypothesis of an endocrine effect as the possible mechanism of action of RYGB.

(Ann Surg 2004;240: 236-242)

S urgery represents the most effective therapeutic modality for morbid obesity. ${ }^{1,2}$ Interestingly, the Roux-en-Y gastric bypass (RYGB), a procedure that includes the creation of a small proximal gastric pouch and the bypass of the duodenum and proximal jejunum from the transit of food, not only determines permanent reduction of excess body weight, but also induces resolution of type 2 diabetes mellitus in more than $80 \%$ of morbidly obese patients. ${ }^{3}$

Body weight is the result of complex physiologic mechanisms that control food intake and energy expenditure. Regulation of body weight involves several systems such as the adipose tissue, ${ }^{4}$ the hypothalamus, ${ }^{5}$ and the adrenal glands, ${ }^{6}$ which are linked by neural and hormonal signals. Recent investigations have identified several key molecules that regulate food intake, including ghrelin, neuropeptide $\mathrm{Y}$ (NPY), ${ }^{7}$ and melanocortins ${ }^{8}$ and have focused on the relationship between obesity and leptin levels. ${ }^{9}$

The effectiveness of gastric bypass in inducing remarkable weight loss and control of diabetes represents an "experimental model" for scientific investigations on the effects and regulation of several gastrointestinal hormones as well as an opportunity to possibly identify the pathogenetic mechanisms underlying obesity and type 2 diabetes mellitus. Several studies have investigated the hormonal changes that follow morbid obesity surgery. In most studies, however, tests are performed after significant weight reduction has occurred, and therefore, it is not possible to establish whether the observed hormonal effect is the cause or the consequence of weight loss and diabetes control. 
The purpose of this study was to evaluate the early effect of gastric bypass on hormones involved in body weight regulation and glucose metabolism, prior to the occurrence of significant weight loss.

\section{MATERIALS AND METHODS}

The study was approved by the Institutional Review Board of the Mount Sinai Medical Center of New York, NY. Ten morbidly obese patients gave informed consent and were included in the study. Table 1 shows the inclusion and exclusion criteria used for the recruitment of patients.

\section{Patients}

There were 9 women and 1 man. The mean age was 36.9 years (range $23-55$ years). The mean preoperative body mass index (BMI) was $46.2 \mathrm{~kg} / \mathrm{m}^{2}$ (range $40-53 \mathrm{~kg} / \mathrm{m}^{2}$ ). Six patients had type 2 diabetes mellitus and were on oral hypoglycemic agents. Other comorbidities are reported in Table 2.

\section{Hormones Measured}

Preoperatively and 3 weeks, postoperatively, the patients were tested for fasting glucose, insulin, glucagon, insulin-like growth factor 1 (IGF-1), leptin, gastric inhibitory polypeptide (GIP), glucagon-like peptide-1 (GLP-1), cholecystokinin (CCK), ACTH, corticosterone, and neuropeptide Y (NPY).

TABLE 1. Inclusion and Exclusion Criteria for Roux-en-Y Gastric Bypass

Inclusion Criteria
BMI of $40 \mathrm{~kg} / \mathrm{m}^{2}$ or greater
BMI of $35 \mathrm{~kg} / \mathrm{m}^{2}$ or greater with comorbidities
No contraindications for surgery as determined by
multidisciplinary bariatric surgery team
Between 18 and 60 yrs of age
Able to provide informed consent
Exclusion Criteria
Patient enrolled in another clinical study involving an
$\quad$ investigational drug or other surgical procedures
Any condition where protocol compliance was unlikely (e.g.,
anxiety disorder, inadequate comprehension)
Pregnancy, immunosuppressive drugs including corticosteroids
Coagulopathy (INR $>1.5$ or platelets $<50$ )
Anemia (Hgb $<10$ )
Any contraindication to RYGB
A significant malabsorptive or gastrointestinal disorder
(pancreatic insufficiency, Celiac Sprue, Crohn disease)
Recent history of cancer ( $<5$ yrs), myocardial infarction in the
previous year, current angina or heart failure

BMI, body mass index; INR, international normalized ratio; Hgb, hemoglobin; RYGB, Roux-en-Y gastric bypass.

\begin{tabular}{lccccc}
\hline TABLE 2. & \multicolumn{5}{l}{ Patient Data } \\
$\begin{array}{lcccc}\text { Patient } \\
\text { No. }\end{array}$ & Gender & $\begin{array}{c}\text { Age } \\
\text { (yrs) }\end{array}$ & $\begin{array}{c}\text { Preop } \\
\text { BMI }\end{array}$ & $\begin{array}{c}\text { Postop } \\
\text { BMI }\end{array}$ & Comorbidities \\
\hline 1 & F & 41 & 44 & 42 & D, hT \\
2 & F & 25 & 46.7 & 43 & JP \\
3 & M & 39 & 50 & 47 & D, jP \\
4 & F & 23 & 53 & 50 & JP \\
5 & F & 35 & 48 & 45 & D, hT \\
6 & F & 31 & 43 & 39 & D, hT \\
7 & F & 37 & 46 & 43 & D, jP \\
8 & F & 55 & 40 & 38 & JP, sA \\
9 & F & 33 & 41 & 37 & JP \\
10 & F & 50 & 51 & 48 & D \\
\hline
\end{tabular}

$\mathrm{D}$, diabetes (pharmacological therapy); Ht, hypertension; JP, joint pain; $\mathrm{Sa}$, sleep apnea.

\section{Surgical Procedure}

All patients underwent laparoscopic RYGB. The surgical technique was as follows: a small proximal gastric pouch of about $20 \mathrm{~mL}$ was created with several firings of a linear stapling device, the jejunum was divided $50 \mathrm{~cm}$ distal to the ligament of Treitz, and an end-to-side gastrojejunostomy was performed by using a $25-\mathrm{mm}$ circular stapler. A side-to-side jejunojejunostomy was then created, $100 \mathrm{~cm}$ distal to the gastrojejunostomy.

At the first ambulatory visit, 3 weeks following surgery, all hormonal tests were repeated under the same fasting condition, and postoperative BMI was also calculated.

\section{Laboratory Tests Analytical Methods}

Blood for plasma glucose was collected in tubes containing sodium fluoride and was measured colorimetrically using an automated glucose oxidase procedure (Vitros, 950 AT, Johnson and Johnson, Rochester, NY). Serum insulin was determined using a validated automated immuno-metric assay (Immulite, Diagnostic Products Corp, Los Angeles, CA).

Specimens for ACTH were collected in tubes containing ethylenediaminetetraacetic acid (EDTA) and immediately put on ice, centrifuged at $4{ }^{\circ} \mathrm{C}$, and stored at $-20^{\circ} \mathrm{C}$ until assayed. Plasma ACTH was measured using a commercially available immunoradiometric assay (Nichols Institute Diagnostics, San Juan Capistrano, CA). Plasma glucagon, serum corticosterone, IGF-1, and leptin were assayed by Endocrine Sciences Inc. (Calabasas, CA), using double antibody radioimmunoassays for glucagons, IGF-1, and leptin. Ammonium sulfate separation was used in the corticosterone assay. Samples were placed on ice after collection and were frozen immediately after separation and stored at $-20^{\circ} \mathrm{C}$ until assayed. 


\section{Preoperative GIP}

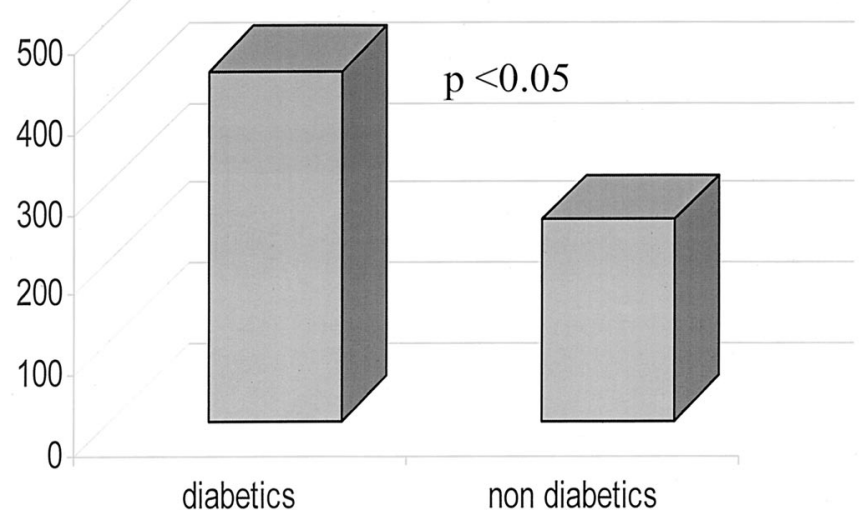

FIGURE 1. Preoperative values of GIP in diabetic and nondiabetic patients.

Cholecystokinin, GLP, GIP, and NPY were assayed by Inter Science Institute (Inglewood, CA) using double antibody radioimmunoassay procedures. Blood for these assays was collected in tubes containing EDTA and Trasylol, an inhibitor of proteolysis. Samples were immediately placed on ice, centrifuged at $4{ }^{\circ} \mathrm{C}$, and the plasma stored at $-20^{\circ} \mathrm{C}$ until assayed.

\section{Statistics}

Statistical analysis was performed with Student paired $t$ test and the Wilcoxon signed ranks test; statistical comparison between the subgroups of diabetic and nondiabetic patients were performed by using 2-tailed unpaired $t$ test.

\section{RESULTS}

All 10 patients underwent successful laparoscopic gastric bypass, with no conversion to open surgery or intraoperative and postoperative complications. They were all discharged on the third postoperative day.

Three weeks after surgery, the mean BMI was not yet significantly changed $\left(43.2 \mathrm{~kg} / \mathrm{m}^{2}\right.$ versus $46.2 \mathrm{~kg} / \mathrm{m} ;{ }^{2} P=$ not significant), whereas blood glucose $(P=0.005)$, insulin $(P=$ $0.02)$, IGF-1 $(P<0.05)$, and leptin levels $(P=0.001)$ were significantly reduced compared to their preoperative values. Mean ACTH increased from $15.38 \pm 8.3 \mathrm{pg} / \mathrm{mL}$ to $20.99 \pm$ $5.0 \mathrm{pg} / \mathrm{mL}(P=0.01)$. There was also an increase in the levels of enteroglucagon, corticosterone, GLP-1, and CCK and a decrease of GIP and NPY, although these changes did not reach statistical significance.

The 6 patients with type 2 diabetes mellitus were euglycemic without medications within 3 weeks of surgery. Insulin levels also returned to normal. Diabetic patients had preoperative levels of GIP higher than normal range for humans. The GIP levels in patients with diabetes were also significantly higher compared with those of the obese nondiabetic patients of our study population (Fig. 1). After surgery, GIP decreased to normal values in diabetic patients $(P<$ 0.01 ), whereas it increased slightly but not significantly in obese nondiabetics (Fig. 2).

Results of blood glucose and hormonal levels are reported in detail in Table 3.

\section{DISCUSSION}

It is commonly believed that the RYGB induces weight loss through a combination of mechanisms, including decreasing the capacity of the stomach, restricting the rate of emptying of the pouch, prohibiting the intake of high osmolar foods, and causing selective malabsorption of fat. Moreover, by enabling salivary secretions and meals going directly into the distal jejunum, RYGB might stimulate the release of gut hormones, which inhibit the brain appetite center or cause the feeling of nausea. ${ }^{10}$ The observation that RYGB induces resolution of type 2 diabetes mellitus prior to the occurrence of weight loss ${ }^{11}$ further suggests that the mechanism of action of this operation might involve some endocrine effects.

We acknowledge several limitations of this study. First, testing only the basal hormonal levels might have overlooked other significant effects of gastric bypass on the meal-induced hormonal secretion. Indeed, many gastrointestinal hormones are stimulated/inhibited after meals, and alterations of the dynamic of this response are likely to occur after bypassing the duodenum and the proximal jejunum. Furthermore, we must admit that a control group of patients matched for BMI and undergoing other interventional procedures would have strengthen the conclusion that the observed hormonal changes are specific of the RYGB. However, the same find-

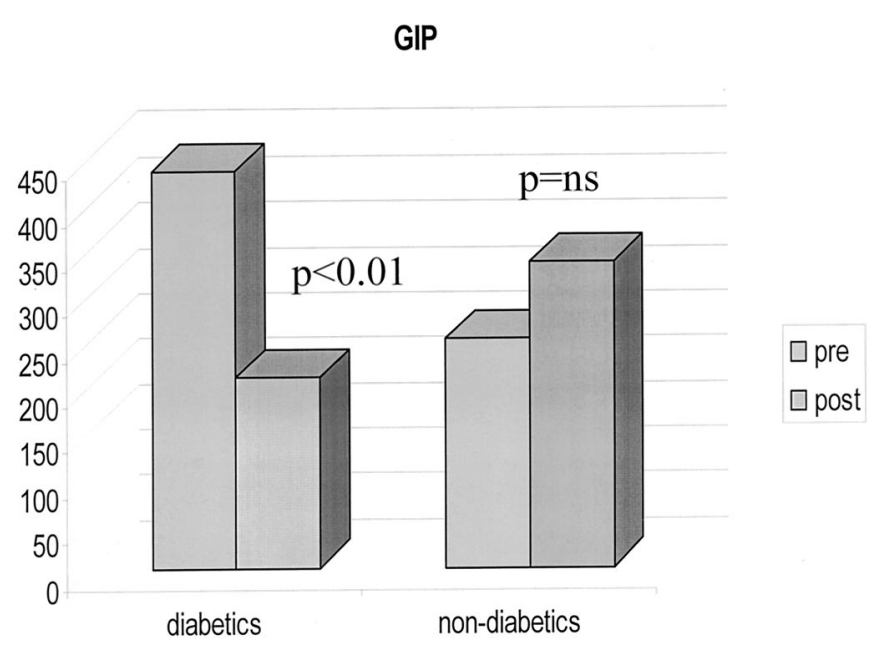

FIGURE 2. Different effect of gastric bypass on GIP in diabetic and nondiabetic patients. 
TABLE 3. Blood Glucose and Hormone Level Results

\begin{tabular}{lccl}
\hline Test & Mean Preop Value & Mean Postop Value & $P$ \\
\hline Glucose & $113.7 \pm 23.3 \mathrm{mg} / \mathrm{dL}$ & $84.8 \pm 5.5 \mathrm{mg} / \mathrm{dL}$ & $0.005^{*}$ \\
Insulin & $24.6 \pm 17.3 \mu \mathrm{IU} / \mathrm{mL}$ & $12.6 \pm 11.7 \mu \mathrm{IU} / \mathrm{mL}$ & $0.01^{*}$ \\
Leptin & $41.6 \pm 19.2 \mathrm{ng} / \mathrm{mL}$ & $19.6 \pm 19.7 \mathrm{ng} / \mathrm{mL}$ & $0.001^{*}$ \\
IGF-1 & $140.6 \pm 13.0 \mathrm{ng} / \mathrm{mL}$ & $110.8 \pm 37.3 \mathrm{ng} / \mathrm{mL}$ & $0.03^{*}$ \\
ACTH & $15.38 \pm 8.3 \mathrm{pg} / \mathrm{mL}$ & $20.99 \pm 5.0 \mathrm{pg} / \mathrm{mL}$ & $0.01^{*}$ \\
Glucagon & $65.8 \pm 30.0 \mathrm{pg} / \mathrm{mL}$ & $70.1 \pm 30.5 \mathrm{pg} / \mathrm{mL}$ & 0.7 \\
Corticosterone & $296.5 \pm 117.8 \mathrm{ng} / \mathrm{dL}$ & $391.4 \pm 170.9 \mathrm{ng} / \mathrm{dL}$ & 0.09 \\
CCK & $15.7 \pm 20.1 \mathrm{pg} / \mathrm{mL}$ & $11.32 \pm 2.7 \mathrm{pg} / \mathrm{mL}$ & 0.5 \\
GIP & $363.4 \pm 169.8 \mathrm{pg} / \mathrm{mL}$ & $260.1 \pm 172.1 \mathrm{pg} / \mathrm{mL}$ & 0.1 \\
GLP-1 & $62 \pm 23.4 \mathrm{pg} / \mathrm{mL}$ & $89 \pm 46.7 \mathrm{pg} / \mathrm{mL}$ & 0.1 \\
NPY & $1.14 \pm 0.84 \mathrm{ng} / \mathrm{mL}$ & $0.81 \pm 0.11 \mathrm{ng} / \mathrm{mL}$ & 0.2 \\
\hline \multicolumn{4}{c}{ *Statistically significant $(P<0.05)}$. \\
\multicolumn{4}{c}{ IGF-1, insulin-like growth factor-1; ACTH, adrenocorticotropic hormone; CCK, cholecysto- } \\
kinin; GIP, gastric inhibitory polypeptide; GLP-1, glucagon-like peptide-1; NPY, neuropeptide Y. \\
\hline
\end{tabular}

ings of our study suggest that the latter limitation might not have caused significant bias to our results. Indeed, we should expect surgical stress to cause alterations of glucose metabolism that go exactly in the opposite direction of the one that we have seen in our study. For instance, insulin levels and plasma glucose should increase under stress conditions, whereas we found that they significantly fall after RYGB.

In spite of the above-mentioned limitations, our findings have implications for the identification of the mechanism of action of RYGB. Indeed, unlike our study, previous investigations on the hormonal effect of RYGB and other bariatric procedures do not clearly rule out the confounding influence of weight loss as a source of hormones variability. Commonly, these reports are based on hormonal tests performed months or years after surgery, when many if not most patients have already experienced substantial weight loss and have BMI levels that are no longer in the range of morbid obesity. Stating that any hormonal or metabolic change is involved in the mechanism of action of a certain bariatric procedure requires the demonstration that these effects occur before that significant weight loss and changes in body composition occur.

We think that our study protocol could represent a valuable model for researches investigating the mechanism of action of bariatric procedures. Indeed, we anticipated that the 3 postoperative weeks could be a reasonable period of time for the patients to recover from the surgical stress, yet not long enough for substantial weight loss and changes in BMI to take place. This was confirmed by our findings documenting rather minimal and not significant variation of mean BMI, which remained, by all means, within the range of morbid obesity during the study's period.

Data from our study, therefore, support the hypothesis that RYGB has an endocrine mechanism of action.
However, due to the lack of knowledge on the exact molecular mechanisms causing insulin resistance and related conditions, whether and which hormonal changes we observed can be responsible for the resolution of obesity and diabetic abnormalities after RYGB remains unanswered. Nevertheless, as the substances tested in our study have important functions in the complex physiologic regulation of body weight and glucose metabolism and significant alterations of their levels or activity have been consistently reported among patients with obesity or type 2 diabetes mellitus, the possibility of their involvement in the mechanism of action of RYGB is quite suggestive.

\section{The Role of Leptin}

Leptin, is an adipocyte-derived hormone. In mice, leptin acts as a hormonal signal in the afferent limb of a negative feedback loop between the adipose tissue and hypotalamic centers. ${ }^{7}$ Upon deposition of fat, plasma leptin level rises and stimulate hypothalamic leptin receptors, ${ }^{12}$ causing a decrease of neuropeptide Y levels in the ventromedial nucleus, eventually resulting in appetite reduction and simultaneous increase in metabolic rate. ${ }^{13,14}$ Physiological increase in plasma leptin has been shown to significantly inhibit glucose-stimulated insulin secretion in vivo and to determine insulin resistance. ${ }^{15}$

Plasma leptin concentration usually reflects the total amount of fat present in the body, and lower plasma leptin levels have been reported consistently among weight-losing patients. ${ }^{16,17}$ The results of our study show that significant reduction of leptin levels occur early after gastric bypass when BMI is still in the morbidly obese range. This is consistent with previous knowledge. Hickey et al, in fact, documented lower levels of leptin in patients undergoing gastric bypass despite stable BMI with respect to a control 
group of morbidly obese patients matched for BMI but not undergoing surgery. ${ }^{11}$ These data suggest that leptin levels might be regulated by mechanisms influenced by the gastric bypass and not solely reflect the body fat content. The normalization of leptin levels, which may reflect a surgically induced improvement of leptin sensitivity, may play a role, directly or indirectly, in the induction of weight loss and diabetes control after RYGB.

\section{The Role of Adrenocorticotropic Hormone}

The novel interesting data of our study are that ACTH levels are significantly increased after RYGB along with a concomitant increase of corticosterone levels. The lack of a control group of surgical patients undergoing other operative procedures does not allow us to conclude whether the observed increase in ACTH levels simply reflects surgical stress or is a specific effect of the gastric bypass. However, it is unlikely that surgical stress alone may induce persistent high levels of ACTH for more than 2 weeks. Indeed, in rats, ACTH returns to basal levels 24 hours after laparotomy, ${ }^{18}$ and normalization of ACTH and cortisol values has been shown to occur within 24 hours in a prospective cohort of 78 children undergoing elective or emergency surgery. ${ }^{19}$

Changes in ACTH have potential significance for the mechanism of action of RYBG. Recent studies indeed demonstrated abnormalities of the hypothalamic-pituitary-adrenal axis (HPA) in central obesity. Obese patients with central obesity exhibit hyperactivity of the HPA axis as reflected by an exaggerated $\mathrm{ACTH}$ response to corticotropin-releasing hormone (CRH) stimulation. ${ }^{20}$ Leptin inhibits stress-responsive secretion of hypothalamic $\mathrm{CRH}$ in mice ${ }^{21}$ and also regulates adrenal function directly, decreasing steroid hormone release in all 3 zones of the adrenal cortex. ${ }^{22}$

One possible explanation for the increased ACTH levels after RYGB could be that the decreased leptin levels after surgery reduce the tonic inhibitory effect of this hormone on the HPA axis. Alternatively, increased ACTH levels may reflect the activation of the pro-opiomelanocortin gene. Adrenocorticotropic hormone, in fact, derives from the same precursor as alpha-MSH, named pro-opiomeanocortin (POMC), which plays an important role in the regulation of appetite and energy expenditure. ${ }^{5,8,23}$ Both the NPY and the POMC systems receive information about the nutritional status and the level of energy storage through insulin and leptin signaling mediated by specific receptors located in the arcuate nucleus. ${ }^{5}$ It is possible that hormonal or neural signals originating from the bowel can regulate expression of the POMC gene either directly or via leptin and insulin signaling. This would result in increased alpha-MSH levels that might explain the loss of desire to eat characteristic of patients undergoing gastric bypass. In this scenario, increased ACTH levels could just be a secondary effect of the stimulation of POMC gene, just as in Addison disease, increased production of ACTH leads to a secondary increase of MSH, responsible for the characteristic pigmentation. Researches investigating the effect of RYGB on alpha-MSH are needed to confirm this hypothesis.

\section{The Control of Diabetes After Roux-en-Y Gastric Bypass}

Experience with surgery for morbid obesity shows that both the gastric bypass and the biliopancreatic diversion not only induce significant and durable weight loss, but also produce amelioration or resolution of comorbid disease states, in particular type 2 diabetes mellitus. ${ }^{24,25}$ Several series show that RYGB normalizes hyperglycemia, ${ }^{3,26,27}$ restores insulin sensitivity, ${ }^{11,28}$ prevents progression from impaired glucose tolerance to diabetes, ${ }^{29}$ and possibly reduces mortality from diabetes mellitus. ${ }^{30}$ Most series report return to euglycemia and normal insulin within days after surgery, long before there is any significant weight loss, ${ }^{3,27}$ and this suggests that the control of diabetes is probably mediated by changes in hormones secretion from the gastrointestinal tract.

Consistent with what has been reported by others, all patients with diabetes in our study experienced rapid normalization of blood glucose and insulin levels and did not require medications within the time frame of our postoperative observation.

Whereas rapid normalization of insulin levels is an already known effect of RYGB, a new interesting finding of our study is the effect on glucose-dependent insulinotropic polypeptide (GIP) levels in diabetic patients. First, we found higher than normal GIP levels in diabetic patients before surgery, whereas in nondiabetic patients, GIP levels were well within the normal range, the difference between the 2 groups being statistically significant (Fig. 1). Surprisingly, RYGB decreased GIP levels in diabetic patients but not in obese nondiabetics (Fig. 2). Admittedly, the relatively small number of diabetic patients (6) in our study population recommends caution in extrapolating firm conclusions from our observation. Nevertheless, the crucial role that GIP plays in glucose metabolism and type 2 diabetes makes these findings noteworthy.

GIP is synthesized and released in the duodenum and proximal jejunum especially in response to glucose and fat ingestion. It acts as an insulinotropic agent with a stimulatory effect on insulin release and synthesis. ${ }^{31}$ Defects in its signaling pathways are considered among the most critical alterations underlying type 2 diabetes, in which the incretin effect of GIP is characteristically attenuated, ${ }^{17}$ secondary to decreased expression of glucose-dependent insulinotropic polypeptide receptor (GIPR). ${ }^{32}$

We speculate that in susceptible individuals, chronic exaggerated stimulation of the proximal gut with fat and carbohydrates may induce overproduction of an unknown factor that cause impairment of GIPR expression or GIP/ 
GIPR interaction, leading to insufficient or inappropriately timed insulin secretion so that glucose intolerance develops. Accordingly, the elevated GIP levels observed preoperatively in our subgroup of diabetic patients may be the result of an attempt to overcome this "GIP-resistant state." It is possible that the duodenal-jejunal exclusion characteristic of the RYGB resolves this aberration, re-establishing normal GIP sensitivity and thus reducing circulating GIP levels.

Some have suggested that greater production of GLP-1, triggered by the earlier presentation of undigested food in lower segments of the bowel, might be involved in the glycemic control consequent to bypass procedures for obesity surgery. Unlike GIP, GLP-1 is in fact mostly produced by $\mathrm{L}$-cells in the distal bowel ${ }^{33}$ and has been shown to significantly improve or even restore normal glucose-induced insulin secretion in diabetic patients. ${ }^{34}$ Although changes in GLP-1 levels did not reach statistical significance in our study, possibly due to the small sample size, we did observe increased GLP-1 levels after surgery, and this may have played an additional role in the resolution of type 2 diabetes after RYGB.

\section{CONCLUSION}

Our study shows that RYGB induces significant changes of the levels of hormones involved in the regulation of body weight and glucose metabolism in the early postoperative period. Our study supports the hypothesis of an endocrine effect as the mechanism of action of the operation, as the observed hormonal changes anticipated the occurrence of significant BMI modifications. The observation that RYGB decreases GIP levels only in patients with diabetes but not in nondiabetic obese patients deserves further investigation, as this may possibly explain how RYGB controls type 2 diabetes, as well as lead to a better understanding of the role of this hormone in the pathogenesis of the disease.

We consider RYGB not only as an effective therapeutic modality for the treatment of morbid obesity, but also as an "experimental model" to improve our knowledge on the etiology of obesity and type 2 diabetes whose epidemic proportions represent one of the greatest challenges to health care systems in the decades to come.

\section{REFERENCES}

1. Buchwald H. Overview of bariatric surgery. J Am Coll Surg. 2002;194: 367-375.

2. Brolin RE. Update: NIH consensus conference. Gastrointestinal surgery for severe obesity. Nutrition. 1996;12:403-404.

3. Pories WJ, Swanson MS, MacDonald KG, et al. Who would have thought it? An operation proves to be the most effective therapy for adult-onset diabetes mellitus. Ann Surg. 1995;222:339-350.

4. Fruhbeck G, Gomez-Ambrosi J, Muruzabal FJ, et al. The adipocyte: a model for integration of endocrine and metabolic signaling in energy metabolism regulation. Am J Physiol Endocrinol Metab. 2001;280: E827-E847.

5. Beck B. Neuropeptides and obesity. Nutrition. 2000;16:916-923.

6. Sainsbury A, Cusin I, Rohner-Jeanrenaud F. Adrenalectomy prevents the obesity syndrome produced by chronic central neuropeptide Y infusion in normal rats. Diabetes. 1997;46:209-214.

7. Rohner-Jeanrenaud F, Cusin I, Sainsbury A, et al. The loop system between Neuropeptide Y and Leptin in normal and obese rodents. Horm Metab Res. 1996;28:642-648.

8. Vergoni AV, Bertolini A. Role of melanocortins in the central control of feeding. Eur J Pharmacol. 2000;405:25-32.

9. Mantzoros CS. The role of leptin in human obesity and disease: a review of current evidence. Ann Intern Med. 1999;130:671-680.

10. Fobi MA, Lee H, Holness R, et al. Gastric bypass operation for obesity. World J Surg. 1998;22:925-935.

11. Hickey MS, Pories WJ, MacDonald KG, et al. A new paradigm for type 2 diabetes mellitus: could it be a disease of the foregut? Ann Surg. 1998;227:637-643; discussion 643-644.

12. Campfield LA, Smith FJ, Guisez Y, et al. Recombinant mouse OB protein: evidence for a peripheral signal linking adiposity and central neural network. Science. 1995;269:546-549.

13. Hwa JJ, Fawzi AB, Graziano MP, et al. Leptin increases energy expenditure and selectively promotes fat metabolism in ob/ob mice. Am J Physiol. 1997;272:R1204-R1209.

14. Pelleymounter MA, Cullen MJ, Baker MB, et al. Effects of the obese gene product on body weight regulation in ob/ob mice. Science. 1995; 269:540-543.

15. Cases JA, Gabriely I, Ma XH, et al. Physiological increase in plasma leptin markedly inhibits insulin secretion in vivo. Diabetes. 2001;50: 348-352.

16. Wallace AM, Sattar N, McMillan DC. Effect of weight loss and the inflammatory response on leptin concentrations in gastrointestinal cancer patients. Clin Cancer Res. 1998;4:2977-2979.

17. SchSchols AM, Creutzberg EC, Buurman WA, et al. Plasma leptin is related to proinflammatory status and dietary intake in patients with chronic obstructive pulmonary disease. Am J Respir Crit Care Med. 1999;160:1220-1226.

18. DeKeyser FG, Leker RR, Weidenfeld J. Activation of the adrenocortical axis by surgical stress: involvement of central norepinephrine and interleukin-1. Neuroimmunomodulation. 2000;7:182-188.

19. Castejon-Casado J, Moreno-Prieto M, Valladares-Mendias JC, et al. Hormonal response to surgical stress in schoolchildren. Eur J Pediatr Surg. 2001;11:44-47.

20. Solano MP, Kumar M, Fernandez B, et al. The pituitary response to ovine corticotropin-releasing hormone is enhanced in obese men and correlates with insulin resistance. Horm Metab Res. 2001;33:39-43.

21. Heiman ML, Ahima RS, Craft LS, et al. Leptin inhibition of the hypothalamic-pituitary-adrenal axis in response to stress. Endocrinology. 1997;138:3859-3863.

22. Glasow A, Bornstein SR. Leptin and the adrenal gland. Eur J Clin Invest. 2000;30(suppl 3):39-45.

23. Vaisse C, Clement K, Durand E, et al. Melanocortin-4 receptor mutations are a frequent and heterogeneous cause of morbid obesity. $J$ Clin Invest. 2000;106:253-262.

24. Smith S, Edwards CB, Goodman GN. Changes in diabetic management after Roux en-Y gastric bypass. Obes Surg. 1996;6:345-348.

25. Cowan GS, Buffington CK. Significant changes in blood pressure, glucose, and lipids with gastric bypass surgery. World J Surg. 1998;22: 987-992.

26. Marceau P, Hould FS, Simard S, et al. Biliopancreatic diversion with duodenal switch. World J Surg. 1998;22:947-954.

27. Scopinaro N, Adami GF, Marinari GM, et al. Biliopancreatic diversion. World J Surg. 1998;22:936-946.

28. Castagneto M, De Gaetano A, Mingrone G, et al. Normalization of insulin sensitivity in the obese patient after stable weight reduction with biliopancreatic diversion. Obes Surg. 1994;4:161-168.

29. Pories WJ, MacDonald KG, Morgan J, et al. Surgical treatment of obesity and its effect on diabetes: 10-year follow-up. Am J Clin Nutr. 1992;55(suppl):582S-585S.

30. MacDonald KG, Long SD, Swanson MS, et al. The gastric bypass operation reduces the progression and mortality of non-insulin-dependent diabetes mellitus. J Gastrointest Surg. 1997;1:213-220.

31. Pederson RA. Gastric inhibitory polypeptide. In: Walsh JH, Dockray GJ, 
eds. Gut Peptides: Biochemistry and Physiology. New York, NY: Raven Press; 1993;217-259.

32. Miyawaki K, Yamada Y, Yano H, et al. Glucose intolerance caused by a defect in the entero-insular axis: a study in gastric inhibitory polypeptide receptor knockout mice. Proc Natl Acad Sci U S A. 1999;96:1484314847.
33. Drucker DJ. Minireview: the glucagon-like peptides. Endocrinology. 2001;142:521-527.

34. Nauck MA, Heimesaat MM, Orskov C, et al. Preserved incretin activity of glucagon-like peptide 1 [7-36 amide] but not of synthetic human gastric inhibitory polypeptide in patients with type-2 diabetes mellitus. J Clin Invest. 1993;91:301-307. 